\title{
Rheum to Diagnosis: A Mixed Methods Study to Uncover Impediments to Accurate Diagnosis of Nonradiographic Axial Spondyloarthritis in the United States
}

\section{Sonam Kiwalkar ( $\nabla$ skiwalkar@tvc.org )}

Vancouver Clinic https://orcid.org/0000-0002-9507-9617

\section{Richard Howard}

Spondylitis Association of America

\section{Dongseok Choi}

Oregon Health \& Science University

\section{Atul Deodhar}

Oregon Health \& Science University

\section{Research article}

Keywords: axial spondyloarthritis, nonradioagraphic axial spondyloarthritis, ankylosing spondylitis, inflammatory back pain, diagnosis, sacroiliac joint, disparities, nr-axSpA, axSpA, SpA

Posted Date: September 15th, 2021

DOI: https://doi.org/10.21203/rs.3.rs-876767/v1

License: (c) (i) This work is licensed under a Creative Commons Attribution 4.0 International License. Read Full License 


\section{Abstract}

\section{Background}

Delayed diagnosis of axial spondyloarthritis (axSpA) is well documented, but little is known about the distinct diagnostic journey and impediments for US patients with nonradiographic axSpA (nr-axSpA).

\section{Methods}

Interviews of adults with rheumatologist-diagnosed nr-axSpA and of rheumatologists explored the diagnostic journey and barriers to diagnosis. Emerging themes were further explored in an online patient survey. Patients were recruited through Spondylitis Association of America outreach and patient panels.

\section{Results}

Interviews were conducted with 25 patients (mean age, 45 years; $80 \%$ female) and 16 rheumatologists. Survey responses from 186 eligible patients (median age, 40 years; $67 \%$ female) were analyzed. Among surveyed patients, median time from symptom onset to nr-axSpA was 3.25 years. In multivariable analysis, delayed diagnosis was significantly more likely for women and people in rural areas. Most patients consulted $\geq 4$ different types of healthcare providers (HCPs) before seeing a rheumatologist and consulted $\geq 2$ rheumatologists before nr-axSpA diagnosis.

Identified impediments to timely diagnosis included the insidious onset of bothersome but tolerable chronic pain; episodic rather than persistent symptom patterns that seemed related to activity; symptoms other than chronic lumbosacral back pain that were dominant and required medical consultation; and widespread unfamiliarity with and misperceptions about nr-axSpA among frontline HCPs, radiologists, and rheumatologists.

\section{Conclusions}

Delayed nr-axSpA diagnosis is common and reflects HCP knowledge gaps as well as frequent patient presentation with dominant nonaxial symptoms. Targeted HCP education, further research into early disease patterns, and interventions sensitive to the broader spectrum of nr-axSpA manifestations are needed to improve timely diagnosis.

\section{Trial Registration}

Not applicable.

\section{Background}

Axial spondyloarthritis (axSpA) is a chronic inflammatory disease preferentially involving the sacroiliac (SI) joints and spine. It typically manifests with chronic back, neck, or buttock pain beginning before 45 
years, with features such as morning stiffness and pain that improve with exercise but not rest. AxSpA is frequently accompanied by enthesitis, psoriasis, inflammatory bowel disease, and uveitis.

People with definitive radiographic sacroiliitis are classified as having radiographic axSpA, also known as ankylosing spondylitis (AS). Those without radiographic sacroiliitis but with characteristic features of axSpA plus either human leukocyte antigen B27 (HLA-B27) positivity or magnetic resonance imaging (MRI) evidence of active SI inflammation are classified as having nonradiographic axSpA (nr-axSpA).(1) Disease activity and burden are substantial and comparable for patients in both groups.(2-4)

In the United States, the estimated population prevalence of axSpA in adults is $0.9-1.4 \%$.(5) By contrast, the estimated prevalence of diagnosed axSpA is $0.2 \%$.(6) This discrepancy suggests that most people with axSpA have not been clinically identified. Delays of up to 14 years between symptom onset and axSpA diagnosis have been observed and highlight the challenges of timely recognition.(7) This considerable delay translates into greater functional impairment, lesser quality of life, and substantial healthcare costs.(8)

Detection and diagnosis of axSpA are hindered by the very high prevalence of back pain in the general population.(9) In addition, validated diagnostic criteria and biomarkers that discriminate axSpA from other causes of back pain are currently lacking.(10) However, those with nr-axSpA likely face additional barriers. This entity has been defined relatively recently and remains unfamiliar to many healthcare providers (HCPs). Although nr-axSpA is at least as common in women as in men,(2) lingering misperceptions of AS being a predominantly male disease may diminish the recognition of axSpA in women.(11) At the same time, while a growing number of therapies have been shown to improve outcomes for this patient population,(12-17) delayed diagnosis is a major impediment to timely receipt of effective therapies. Prior studies of diagnostic delay have largely focused on patients with AS or axSpA overall.(18-25) Studies exploring the distinct obstacles to nr-axSpA diagnosis are lacking. The present study addresses this gap and aims to better understand patient experiences and the specific barriers that contribute to delay in nr-axSpA diagnosis in the United States.

\section{Methods}

In order to better understand patient experiences and contributions to delay in nr-axSpA diagnosis, this study used a mixed-methods design with qualitative and quantitative components. The study was approved by the Oregon Health \& Science University institutional review board.

\section{Qualitative Component Patient Interviews}

Adults diagnosed with nr-axSpA by rheumatologists were invited via the January 2020 eSun newsletter of the Spondylitis Association of America (SAA) to share their diagnostic journey during a 45-minute interview. To diversify the sampling frame, participants were also recruited from general population and 
back pain market research panels. Interested respondents completed an online screener to confirm rheumatologist-diagnosed nr-axSpA. Individuals who met the primary eligibility criteria (Table 1) and had previously undergone MRI to evaluate their back or pelvic pain were invited to interview.

Table 1

Patient Eligibility Criteria for Qualitative and Quantitative Study Components

- Age $\geq 18$ years

- United States resident

- Diagnosed with nr-axSpA by a rheumatologist

- Back, hip, or buttock pain $\geq 3$ months

- Back, hip, or buttock pain onset before age 45 years

AND affirmation of:

EITHER

$\geq 3$ of the following 4 criteria:

- Gradually developing pain

o Pain improves with physical activity

o Pain worsens with rest

o Pain causes nighttime awakening

OR

$\geq 1$ of the following 7 criteria:

o Pain significantly improves with nonsteroidal anti-inflammatory drugs

o Heel pain

o Psoriasis

o Inflammatory bowel disease

o Uveitis/iritis

o Unexplained joint pain and swelling

o Unexplained swelling of entire fingers or toes

Eligibility was determined by patients' self-reported responses to a short screening questionnaire (qualitative interviews) or to questions contained in the survey instrument (quantitative component). Screening questions are in Additional file 1.

The semistructured interviews explored patients' sequential experiences and perceptions from initial symptom onset to nr-axSpA diagnosis by a rheumatologist (see Additional file 2). Interviews were 
primarily conducted via GoToMeeting, a web-based conferencing and recording platform that allowed participants to view the interviewer's screen. Interviewers illustrated the diagnostic journey (see Additional

file 3) on a template as participants described their experience. Participants were invited to correct and validate what was documented.

\section{Rheumatologist Interviews}

Board-certified US rheumatologists in the SAA database who treat patients with nr-axSpA were invited to participate in 30-minute interviews through email and telephone outreach in February 2020. The semistructured interviews focused on nr-axSpA in the rheumatologist's practice, diagnosis of nr-axSpA, and strategies to improve the diagnostic process (see Additional file 4).

\section{Interview Team and Analysis}

All interviews and analyses were conducted by 2 masters-level professionals -1 male, 1 female-with expertise in qualitative and quantitative research methods. Interview content was analyzed using a grounded theory approach to identify recurrent themes.

\section{Quantitative Patient Survey Component}

An online patient survey was fielded between April and July 2020 to further explore themes arising from qualitative analysis. Patients were invited to participate and share their nr-axSpA experience via SAA's electronic eSun newsletter (April and May 2020 issues), on social media channels, and through market research databases on the general population and on people with back pain. Initial survey questions assessed respondents' eligibility (see Additional file 1). Subsequent items explored patients' healthcare experiences leading up to and after their initial rheumatology consultation, barriers and facilitators affecting their nr-axSpA diagnosis, and demographic characteristics.

\section{Statistical Analysis}

Time to nr-axSpA diagnosis was a variable calculated from 2 separate, open-ended questions that asked for approximate month and year of symptom onset and of nr-axSpA diagnosis.

A multiple logistic regression analysis of patient survey data was undertaken using $\mathrm{R}$ statistical language (v3.6.3) to evaluate factors that might increase time to nr-axSpA diagnosis. Analyses centered on a new binary variable, delayed diagnosis (defined as greater than, or less than or equal to, median time to nraxSpA diagnosis). The initial model included the following dichotomized independent variables: sex (male vs female), location (rural vs nonrural), race (white vs nonwhite), education (bachelor's degree or higher vs less than a bachelor's degree), work status (employed full-time vs not employed full-time), and income $(<\$ 5,000$ a year vs $\geq \$ 75,000$ a year). Independent variables with no significant association were removed from the final model.

\section{Results}




\section{Patient Interviews}

Interviews were conducted with 24 of 165 respondents recruited from SAA outreach and 1 of 1425 respondents recruited via market research panels (Fig. 1). Mean age of interviewed patients was 45 years, and $80 \%$ were female. Time to nr-axSpA diagnosis was $>6$ years (68\%), 3 to 5 years (24\%), and 1 to 2 years (8\%). All but 3 patients had been treated with biologic therapy, and nearly a third worked in healthcare settings.

\section{Early Symptoms Did Not Necessarily Prompt Medical Attention}

Several barriers were revealed in interviews. Themes and illustrative quotes are shown in Table 2 and the more-detailed Additional file 5. Interviewed patients had diverse experiences with early nr-axSpA symptoms. For some, early symptoms caused pain or functional impairment that was severe enough for them to quickly seek medical attention. Another subset described an insidious background of chronic pain that waxed and waned and was often perceived as normal or an unremarkable wear-and-tear response. Yet others experienced episodic symptom flares. For a few patients, flares seemed random, but more commonly they coincided with exertional activities. Unless symptoms interfered with their activities, young and athletic patients often tolerated these episodes, having learned that flares eventually resolved. 
Table 2

Impediments to nr-axSpA Diagnosis: Themes and Selected Illustrative Quotes From Qualitative Patient Interviews

The insidious onset of early axSpA symptoms leads many patients to perceive their pain as bothersome but not alarming. Patients tolerate it without necessarily seeking medical care.

"My first symptoms started in my late 20s. I just thought it was normal back pain. It started in my SI joints....And what I did was go to a chiropractor. I didn't go to a doctor." (Patient 107)

“I developed back pain in my teen years. It wasn't horrible. It wasn't like the full-on $\nabla$ l can't sleep at

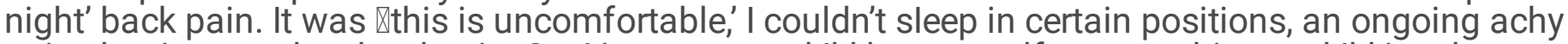
pain...but it was a low-level pain. So, I just assumed l'd hurt myself or something and I'd just have to live with it." (Patient 198)

\section{Patients' chief complaints may not reflect the ASAS axSpA paradigm of insidious and persistent chronic low back pain. Rather, patients' most troubling symptoms often involve the neck or nonaxial sites, flare episodically, or seem associated with strenuous activity or trauma.}

"Starting in middle school and all through high school, I saw an orthopedic doctor for my knees, never my back, cause my knees would always cause a problem-and they could never figure out what the cause was-they said it was probably just growing pains, but my knees would give out on me, be achy all the time." (Patient 211)

"I first starting having issues in high school. My back would go out.... I would be walking into school... and all of a sudden, I would have sciatic pain and I could hardly walk. But it would come and go. And we never really did anything about it." (Patient 103)

"If I'd decided to vacuum...that would have put me out [with back pain] for a few days." (Patient 103)

"All of a sudden my neck is stiff. It didn't occur to me to go to a doctor that first time. And go, खHeymy neck's stiff; let's figure this out.' It was stiff and I thought, well it will go away. And it did....and then it came back, [...but I knew] it went away last time." (Patient 172)

"As a kid, I was slightly conscious of the fact that I would injure myself and the injury would kinda persist longer than it seemed like it should. I remember falling and hitting my heel as a young child of age 7-ish. That heel was sensitive for probably a year or so. I couldn't play basketball because every time I hit my fingers with the ball, my finger joints would swell up." (Patient 172)

"In my senior year of high school, I would have to go to physical therapy before or after soccer games. I don't know why I never thought to not do those things, but I loved soccer and tennis, so I continued to play them. When I would play tennis, I would play my match, and then my doubles partner would have to carry me off the court because I couldn't walk anymore." (Patient 103)

\section{HCPs are often unaware of and have poor understanding of axSpA, hindering detection and interpretation of nr-axSpA manifestations.}

"They were just treating the symptoms and not figuring out what was wrong." (Patient 123)

"I was told everything is normal by 4 specialists." (Patient 167)

"I was told, खYou can't have AS. That is a man's disease."' (Patient 151)

"[The rheumatologist] dismisses AS right away because I am not HLA + and my x-rays show no evidence.....Now I know this is wrong.... and this rheumatologist is teaching this!" (Patient 241)

"I had tested positive for the HLA-B27, but the rheumatologist told me, 『you just need to lose weight and you'll feel better."' (Patient 103) 


\section{The insidious onset of early axSpA symptoms leads many patients to perceive their pain as bothersome but not alarming. Patients tolerate it without necessarily seeking medical care.}

\section{Patients presenting with nr-axSpA symptoms often lack definitive abnormalities that explain their symptoms, leading to counterproductive clinical encounters and skepticism, particularly toward young and female patients.}

"Most orthos-because I was young and female-thought I was making it up." (Patient 167)

[When the $x$-ray result was normal], "The doctor told me, $\triangle$ Your pain can't be that bad."' (Patient 151)

"I got referred to spine and bone specialists. I was about 21 or $22 \ldots$ and they made me feel like a hypochondriac. So, I stopped going and just dealt with it." (Patient 103)

"If a woman has symptoms that can't be explained, they just assume she has emotional problems." (Patient 165)

\section{Patients face numerous barriers to satisfactory rheumatology care and a definitive diagnosis.}

"I was seeing my general family doctor. He fought me pretty hard on referring me. Back in 2002, I requested that he test me for HLA-B27. He fought me pretty hard on that [too], but I threatened to go to another doctor. He finally ran that test, and it came back positive. So in 2012 , he finally set me up with a referral to a rheumatologist."

"[My doctor didn't want to order the test] because I wasn't a guy. Women can't have AS. That's what I was told."

"Part of the problem was that where we lived, we didn't have a rheumatologist. I would have had to travel to see a specialist. We had one finally in 2012."

"My diagnosis changed last year. I've seen 3 rheumatologists, and this last one changed my diagnosis [to nr-axSpA]." (Patient 213)

"At some point I saw just about every rheumatologist in the metropolitan area, because I wasn't getting anywhere. They sort [of] didn't know what to do, and it didn't seem like they knew much about spondylitis." (Patient 156)

ASAS, Assessment of SpondyloArthritis International Society.

Chronic Back Pain, Central to the axSpA Definition,(26) Was Often Not the Dominant Symptom

All interviewed patients reported low back, hip, or SI joint pain at some point; however, for nearly half of this group, peripheral joints, neck pain, enthesitis, uveitis, and gastrointestinal symptoms caused greater distress and were the initial focus of medical care.

\section{Early Medical Encounters Were Often Unsatisfying}

Patients with early disease typically sought medical care for specific troublesome symptoms, which they sometimes found difficult to describe. HCPs typically performed narrowly focused evaluations at initial visits. When physical examination, imaging studies, and laboratory tests were unremarkable, as was common, HCPs frequently offered reassurance and recommendations that patients found unsatisfying. 
To patients returning with persistent pain symptoms, HCPs often expressed skepticism if symptoms could not be medically explained. In particular, patients who were young and/or female were often questioned about the validity of their pain complaints and motivations. Numerous women reported that their symptoms were attributed to stress, depression, pregnancy, or weight. Further, women repeatedly encountered HCPs who believed that axSpA was a "man's disease." This misperception was a significant barrier to HLA-B27 testing and rheumatology referral. Even when women were found to be HLA-B27 positive or received an axSpA diagnosis, many HCPs disputed the diagnosis.

\section{Physicians, Including Rheumatologists, Lack Knowledge About axSpA}

Most patients saw multiple HCPs before axSpA was considered. These HCPs often knew little about spondyloarthritis and did not recognize indications for rheumatology referral. To fill this void, patients often turned to the internet for answers. Although information on axSpA and especially nr-axSpA has been scarce until recently, when found, it empowered patients to communicate more effectively and advocate for testing and rheumatology care.

\section{Frequent Barriers to Effective Rheumatology Care}

Once referred to a rheumatologist, patients often faced long waits for an appointment. Patients encountered wide variation in rheumatologists, and several expressed alarm at their clinician's limited knowledge of nr-axSpA. When researching potential rheumatologists, patients found few clues about the prospective rheumatologist's spondyloarthropathy expertise and frequently consulted multiple rheumatologists before finding a "good" one. The most satisfying rheumatologists were often found at tertiary centers or through SAA.

\section{Rheumatologist Interviews}

Of 190 invited physicians, 26 completed the qualifying questionnaire, and 16 board-certified US rheumatologists were interviewed (Fig. 1); all had been in practice for $\geq 7$ years and cared for multiple people with nr-axSpA. Five rheumatologists were in academic practices; 1 was a pediatric rheumatologist.

\section{Awareness of nr-axSpA Is Low Among Physicians}

Rheumatologists viewed poor recognition of inflammatory back pain (IBP) as a major factor contributing to diagnostic delay, noting that it is commonly missed by primary care physicians (PCPs), orthopedists, pain specialists, and even some rheumatologists. Patient age and sex may also play a role. Young, seemingly healthy patients often lack a sustained PCP relationship and are less skilled in self-advocacy. Women's pain complaints are frequently dismissed as mechanical back pain by PCPs and specialists. However, rheumatologists also sensed rising awareness of nr-axSpA.

\section{Varied and Latent Symptoms of nr-axSpA Hinder Diagnosis}


Although some of the group perceived nr-axSpA diagnosis as straightforward, others described challenges posed by varied clinical presentations or seemingly unrelated symptoms. Understanding often emerged only after several encounters with a patient.

\section{Several Factors Constrain the Utility of Diagnostic Imaging}

Rheumatologists varied in their reliance on diagnostic imaging when evaluating patients for axSpA. They felt that most radiologists were inadequately trained in reading SI joint radiographs and that many rheumatologists lacked local access to experienced musculoskeletal radiologists. Rheumatologists have greater confidence in MRI, but their radiologists often lack key skills in interpreting axSpA features in this modality. Furthermore, less-experienced rheumatologists may not know how to adequately specify their MRI request to evaluate axSpA. Several interviewed rheumatologists had pursued informal training to improve their own MRI skills.

\section{Quantitative Patient Survey Results}

Of those recruited by SAA outreach, 88 of 252 respondents affirmed rheumatologist-diagnosed nr-axSpA and were deemed eligible, while 98 of 16,031 market research panel respondents were found eligible (Fig. 1). The resulting analysis sample $(n=186)$ was primarily female $(67 \%)$, white $(81 \%)$, and college educated (62\%). Median age was 40 years (Table 3 ). At least $85 \%$ of patients had undergone MRI imaging, and nearly $80 \%$ had undergone HLA-B27 testing, with $59 \%$ reporting positive status. 
Table 3

Characteristics of Patients in Quantitative (Survey) Study Component $(\mathrm{N}=186)$

\begin{tabular}{|ll|}
\hline Sex & \\
\hline Female & $67 \%$ \\
\hline Age, years & \\
\hline Median & 40.0 \\
\hline (interquartile range) & $(34.0-51.8$ ) \\
\hline Race & \\
\hline Asian & $4 \%$ \\
\hline Black & $4 \%$ \\
\hline Hispanic & $6 \%$ \\
\hline White & $81 \%$ \\
\hline Multiracial & $3 \%$ \\
\hline Other & $1 \%$ \\
\hline Education & \\
\hline High school or less & $8 \%$ \\
\hline Trade/technical school & $4 \%$ \\
\hline Some college & $26 \%$ \\
\hline Bachelor's degree & $39 \%$ \\
\hline Advanced degree & $23 \%$ \\
\hline Work status (prepandemic) & \\
\hline Student & $5 \%$ \\
\hline Work full-time & $11 \%$ \\
\hline Work part-time & $9 \%$ \\
\hline Stay-at-home parent & \\
\hline Retired & \\
\hline On disability & \\
\hline Unemployed & \\
\hline
\end{tabular}

a Percentages may not total 100 due to rounding. 


\begin{tabular}{|c|c|}
\hline \multicolumn{2}{|l|}{ Sex } \\
\hline \multicolumn{2}{|l|}{ US region } \\
\hline Northwest & $14 \%$ \\
\hline Northeast & $26 \%$ \\
\hline Southwest & $20 \%$ \\
\hline Southeast & $18 \%$ \\
\hline Central & $22 \%$ \\
\hline \multicolumn{2}{|l|}{ Community type } \\
\hline Large city & $28 \%$ \\
\hline Suburb & $38 \%$ \\
\hline Small city or town & $23 \%$ \\
\hline Rural & $11 \%$ \\
\hline \multicolumn{2}{|l|}{ Household income } \\
\hline$<\$ 25000$ & $9 \%$ \\
\hline$\$ 25000$ to $\$ 49999$ & $15 \%$ \\
\hline$\$ 50000$ to $\$ 99999$ & $28 \%$ \\
\hline$\$ 100000$ to $\$ 149999$ & $31 \%$ \\
\hline$\geq \$ 150000$ & $12 \%$ \\
\hline Prefer not to answer & $6 \%$ \\
\hline \multicolumn{2}{|c|}{ MRI to evaluate back, hip, or buttock pair } \\
\hline Yes, back or pelvis & $75 \%$ \\
\hline Yes, site uncertain & $11 \%$ \\
\hline No & $13 \%$ \\
\hline Unsure & $1 \%$ \\
\hline \multicolumn{2}{|l|}{ HLA-B27 status } \\
\hline Positive & $59 \%$ \\
\hline Negative & $22 \%$ \\
\hline Not tested & $13 \%$ \\
\hline
\end{tabular}




\begin{tabular}{|l|}
\hline Sex \\
\hline Unsure $6 \%$ \\
\hline a Percentages may not total 100 due to rounding. \\
\hline
\end{tabular}

Median time from symptom onset to nr-axSpA diagnosis was 3.25 years (interquartile range, 1.5-8.0 years) (Fig. 2). While $49 \%$ of patients received their diagnosis within 2 years of symptom onset, $23 \%$ of patients experienced symptoms for longer than 11 years before their nr-axSpA diagnosis. Mean age at diagnosis was 37 years.

Patients typically sought care from multiple HCPs before seeing a rheumatologist, with $51 \%$ seeing at least 4 different types of clinicians (see Additional file 6). The most common providers seen were PCPs (70\%), chiropractors (43\%), and physical therapists (40\%) (see Additional file 6). Although $59 \%$ of patients received a rheumatology referral for axSpA, $17 \%$ self referred, and $5 \%$ were already seeing a rheumatologist for another condition. Seeing a rheumatologist did not necessarily provide diagnostic clarity or satisfaction. Almost half of patients received their nr-axSpA diagnosis only after consulting multiple rheumatologists (see Additional file 6), and 84\% sought care for their symptoms from 2 or more rheumatologists.

\section{Factors Contributing to Diagnostic Delay}

When asked which of several patient factors were most impactful in preventing timely nr-axSpA diagnosis, patients most frequently pointed to their own initial perceptions that "joint pains were due to activity or injury" (45\%) or that their "back pain was due to aging" (18\%). The two most impactful healthcare factors that hindered nr-axSpA diagnosis were "healthcare providers [who] attributed my symptoms to activity or injury" (22\%), and "healthcare providers [who] thought my symptoms were "in my head"' (18\%) (Fig. 3 and Additional file 7).

Nondiagnostic studies were additional confounding factors, with normal $x$-ray or MRI results (36\%) and negative HLA-B27 status (58\% of patients were known to be HLA-B27 negative) identified as contributing to diagnostic delay. Patients also indicated multiple barriers to seeing a rheumatologist. Thirty-two percent reported that "medical professionals didn't know to refer me to a rheumatologist," $11 \%$ "requested a rheumatology referral [that] was denied," and 25\% reported "limited access to rheumatologists."

Even after getting to a rheumatologist, recognition and diagnosis of nr-axSpA were not always straightforward. Twenty-two percent of patients indicated that "rheumatologist(s) I saw gave me conflicting diagnoses," and 16\% indicated "rheumatologist(s) I saw were unaware of nr-axSpA." Among women, $25 \%$ were told that "nr-axSpA was a 'man's disease"' and cited this misperception as a barrier.

Misdiagnosis was reported by $25 \%$ of patients, with greater frequency among women than men (33\% vs $10 \%)$. Fibromyalgia was the most frequently cited alternative diagnosis and was reported exclusively by 
women (12 of 124).

Facilitators of nr-axSpA Diagnosis

Seeing a rheumatologist who recognizes the signs and symptoms of nr-axSpA facilitates a timely diagnosis, but this scenario was not experienced by most surveyed patients (see Additional file 8). Only $26 \%$ indicated "I was quickly referred to a rheumatologist," and $42 \%$ reported that "the first rheumatologist diagnosed me quickly and confidently." Patients advocating for themselves (45\%), researching the cause and/or disease on their own (44\%), and referring themselves to a rheumatologist (24\%) were factors that facilitated nr-axSpA diagnosis. For $20 \%$ of patients, their family history helped them recognize the need for rheumatology care.

Predictors of nr-axSpA Diagnostic Delay

Multiple logistic regression analysis of patient survey data indicated that race and income had nonsignificant associations with delayed nr-axSpA diagnosis and were removed from the final model. Patients were significantly more likely to have delayed diagnosis (see Additional file 9) if they were female (OR, 3.75; $95 \% \mathrm{Cl}, 1.88-7.69)$, lived in a rural location (OR, 3.56; $95 \% \mathrm{Cl}, 1.20-10.55)$, had completed college (OR 2.87; $95 \% \mathrm{Cl}, 1.38-5.95)$, or did not have full-time employment (OR $2.84 ; 95 \% \mathrm{Cl}$, 1.41-5.71).

\section{Discussion}

To the best of our knowledge, this study is the first to use both qualitative and quantitative methods to evaluate impediments to nr-axSpA diagnosis in the United States.

Median time to nr-axSpA diagnosis was 3.25 years (mean, 7.9 years) among surveyed patients. Although half of patients were diagnosed within 2 years of symptom onset, more than $20 \%$ lived with undiagnosed or misdiagnosed symptoms for more than a decade. Diagnostic delay was significantly greater among patients who were women, lived in rural areas, were highly educated, and were not working full-time. The delay revealed in this study is consistent with the mean diagnostic delay of 6.7 years for patients with axSpA in a recent systematic review.(27)

This study identified additional factors that contribute to delay. A critical finding is HCPs' poor awareness and understanding of axSpA. Patients commonly encountered outdated perceptions of axSpA that biased HCPs against this diagnosis in women and people who were HLA-B27 negative. More than a third of surveyed patients also indicated that seemingly normal imaging studies contributed to diagnostic delay. In 2009, the Assessment of SpondyloArthritis International Society (ASAS) developed classification criteria for axSpA that deliberately recognize that axSpA can be present without definitive radiographic sacroiliitis.(1) However, our findings suggest that many frontline HCPs are not yet familiar with nr-axSpA.

Several factors limit the utility of imaging studies in facilitating axSpA diagnosis. Radiologists often lacked skill in evaluating x-ray and MRI images of the SI joints and likely missed subtle abnormalities. These findings support a recent survey of radiologists demonstrating prevalent knowledge gaps related 
to axSpA imaging.(28) Studies have shown that the bone marrow edema observed in the MRI of the SI joints in axSpA patients is not specific to the disease but can also occur in athletes, postpartum women, and other healthy populations. $(29,30)$ Furthermore, degenerative spinal disease frequently coexists with $\operatorname{axSpA}(31,32)$ and may diminish attention to less-familiar axSpA-related abnormalities, particularly when axSpA is not suspected. These issues highlight the importance of close collaboration between radiologists and rheumatologists.

Symptoms described by study patients reflect the marked heterogeneity of nr-axSpA. Current efforts to increase awareness of axSpA often emphasize chronic IBP, $(19,33,34)$ and for many patients in this study, persistent back pain was an early and dominant symptom. Yet numerous patients did not have this classic symptom pattern (see Additional file 10). Several patients described acute flares of back pain during their teen years and early adulthood. For other patients, the most painful and disabling symptoms occurred at nonaxial sites, consistent with growing evidence that manifestations of axSpA and peripheral spondyloarthropathy have considerable overlap.(35) Given HCPs' limited understanding of spondyloarthritis, it seems unlikely that they would recognize these diverse presentations of axSpA. Thus, axSpA campaigns that were centered on the timely recognition and referral of the patient with IBP may be overly narrow. Rather, it may be important to bring attention to the broader phenomena associated with spondyloarthritis and the common underlying pathophysiology.(36) Doing so may help HCPs better discern axSpA in patients with varied, asynchronous, and seemingly unrelated manifestations.

Study patients and their physicians often perceived a connection between axSpA symptoms and preceding physical activities or trauma. These perceptions led some patients to initially self manage symptoms in expectation of gradual resolution. In clinical settings, these perceptions likely led patients and physicians to narrowly focus on the chief complaint, overlooking other relevant signs or symptoms.

Patients' perceptions that their strenuous activities and axSpA symptoms are linked has been reported in a separate study (37) and may have validity. It is increasingly understood that spondyloarthritis arises, at least in part, from an exaggerated inflammatory response to biomechanical stress in predisposed individuals. $(38,39)$ Several studies have shown a positive association between physically demanding occupations and axSpA progression; $(40,41)$ however, few studies have explored the temporal relationship between axSpA symptoms and exertional activity or minor trauma. Further research into these associations is warranted and may facilitate early axSpA detection.

Women in this study were significantly more likely than men to experience delayed nr-axSpA diagnosis, supporting findings from a recent systematic review.(42) The present study identified several factors that likely contribute to this disparity. For example, women were more likely to indicate that normal imaging studies, negative HLA-B27 status, and HCP perceptions of axSpA as a "man's disease" hindered their diagnosis. Women were also more than 3 times as likely to report that their nr-axSpA symptoms was misdiagnosed, and more than $40 \%$ of women reported their HCP making disparaging remarks about their symptoms. 
These findings are consistent with a study demonstrating striking sex-specific differences in how rheumatologists documented symptoms of patients with axSpA and showing that men were 3 times more likely to receive an axSpA diagnosis at their first rheumatology visit.(24) The authors concluded that HCPs held gender biases that influenced their clinical assessment, thereby affecting time to diagnosis at multiple time points.

Sex-related differences in the experience of pain may likewise contribute to disparate diagnostic delay. A recent study of axSpA pain found that women had significantly higher rates of pain in the cervicothoracic junction and thoracic spine, as well as higher rates of widespread axial and peripheral articular pain.(43) Biological sex differences that affect the brain's response to pain signals are apparent in infancy and may predispose women to more widespread pain.(44) Psychological and cultural factors also impact how men and women respond to and communicate about pain. These factors collectively influence doctor-patient interactions that center on pain. These disparities were demonstrated by Global Assessment pain and disability ratings that were discordantly higher among nearly $60 \%$ of women, but just $40 \%$ of men, compared with their physician's rating.(45) Women's disparate diagnostic journey is not unique to axSpA but reflects a broader phenomenon seen with medically inexplicable chronic pain.(46)

Finally, patients in this study faced numerous barriers to rheumatology care that hindered timely nr-axSpA diagnosis. Even when patients explicitly requested referral, their HCPs often resisted. These obstacles stem in part from HCPs' failure to recognize IBP and other indicators of rheumatological disease; however, local scarcity of rheumatology services can also be a factor.(47) In this study, rural location was independently associated with diagnostic delay, and several regions of the United States are underserved by rheumatologists.(48) Patients in this study were further hampered by the lack of rheumatologists with contemporary knowledge of nr-axSpA, indicating a continuing need for education on this topic. Patients and referring clinicians need mechanisms to more easily identify rheumatologists skilled in spondyloarthritis care. Strategies to improve the timeliness of nr-axSpA diagnosis must also anticipate regional variation in rheumatology access and be tailored accordingly.

Our study has some limitations. We used a convenience sampling approach that recruited almost all of the qualitative study participants, and more than half of survey respondents, through SAA outreach. These individuals were predominantly female and may not be representative of the broader population living with nr-axSpA. To diversify the sample, participants were also recruited from patient panels. This strategy was inefficient and required additional quality checks but increased male and nonwhite survey participation. In both study phases, the determination that respondents had rheumatology-diagnosed nraxSpA and were eligible relied on self-reported survey responses and could not be independently confirmed.

The qualitative study component revealed patterns in early symptoms and care seeking that may significantly influence time to $\mathrm{nr}$-axSpA diagnosis. These findings were not anticipated and thus were not systematically assessed. Further qualitative and quantitative research into patients' preclinical symptoms and experiences is indicated. 


\section{Conclusions}

In this mixed-methods study of impediments to nr-axSpA diagnosis in the US, delayed diagnosis was common. Among surveyed patients, median time to diagnosis was 3.25 years, yet more than $20 \%$ had undiagnosed symptoms for over a decade. Diagnostic delay disproportionately affected women and patients living in rural areas. Barriers to timely diagnosis included patient tolerance for chronic pain, symptoms other than hallmark chronic low back pain prompting care, and episodic symptom flares seemingly related to strenuous activity. Furthermore, HCPs are typically unfamiliar with axSpA or hold outdated perceptions that contribute to delayed diagnostic testing and rheumatology referral, and qualified rheumatologists who understand axSpA are limited in number.

Further research into early nr-axSpA manifestations and associations with physical activity and minor trauma is needed, as well as education to improve knowledge of nr-axSpA and address misperceptions among frontline HCPs, radiologists, and rheumatologists. Interventions that consider the broader spectrum of presentations beyond lumbosacral IBP seem warranted.

\section{Abbreviation}

AS, ankylosing spondylitis

ASAS, Assessment of SpondyloArthritis International Society

axSpA, axial spondyloarthritis

$\mathrm{Cl}$, confidence interval

HCP, healthcare provider

HLA-B27, human leukocyte antigen B27

IBP, inflammatory back pain

MRI, magnetic resonance imaging

nr-axSpA, nonradiographic axSpA

OR, odds ratio

PCP, primary care physician

SAA, Spondylitis Association of America

SI, sacroiliac

\section{Declarations}


Ethics approval and consent to participate

This study was approved by the Oregon Health \& Science University institutional review board (reference \# 00020788).

\section{Consent for publication}

All study participants provided their consent for participation. Consent forms are available upon request.

\section{Availability of data and methods}

The datasets used and analyzed during the current study are available from the corresponding author on reasonable request.

\section{Competing interests}

SK declares that she has no competing interests.

$\mathrm{RH}$ declares that he has the following competing interests:

- Consulting/advisory board: Novartis, GSK

- Stock: AbbVie, Amgen, Bristol Myers Squibb, GSK, Johnson \& Johnson, Lilly, Merck, Novartis, Pfizer, Teva, UCB, Viatris

DC declares that he has no competing interests.

$A D$ declares that he has the following competing interests:

- Consulting/advisory boards: AbbVie, Amgen, Boehringer Ingelheim, Bristol Myers Squibb, Celgene, GSK, Eli Lilly, Janssen, Novartis, Pfizer, UCB

- Research grants: AbbVie, Lilly, Novartis, Pfizer, UCB

\section{Funding}

UCB provided funding to the Spondylitis Association of America in support of this study. UCB did not participate in the survey design, interpretation of results, or manuscript development.

\section{Authors' contributions}

SK was integral in developing the study design, analyzing and interpreting the study datasets, and liaison with the statistician on dataset analysis. $\mathrm{RH}$ was integral in developing the study design, recruitment and enrollment of patients into the study, and analyzing and interpreting study datasets. DC provided statistical analysis on all datasets and verified data accuracy. AD was integral in development of the study design and analyzing and interpreting study datasets. All authors read and approved the final manuscript. 


\section{Acknowledgments}

The authors would like to thank Dr Jill Foster and the Institute for Medical and Nursing Education editorial team for support in developing this manuscript. We would also like to acknowledge Erin Schulz, John Thomas, and the LaunchBox team for their help in conducting and analyzing interviews and the survey and in reporting initial findings.

\section{References}

1. Rudwaleit M, van der Heijde D, Landewe R, Listing J, Akkoc N, Brandt J, et al. The development of Assessment of SpondyloArthritis international Society classification criteria for axial spondyloarthritis (part II): validation and final selection. Ann Rheum Dis. 2009;68(6):777-83.

2. Lopez-Medina C, Ramiro S, van der Heijde D, Sieper J, Dougados M, Molto A. Characteristics and burden of disease in patients with radiographic and non-radiographic axial Spondyloarthritis: a comparison by systematic literature review and meta-analysis. RMD Open. 2019;5(2):e001108.

3. López-Medina C, Molto A, Claudepierre P, Dougados M. Clinical manifestations, disease activity and disease burden of radiographic versus non-radiographic axial spondyloarthritis over 5 years of follow-up in the DESIR cohort. Ann Rheum Dis. 2020;79(2):209-16.

4. Mease PJ, Heijde DV, Karki C, Palmer JB, Liu M, Pandurengan R, et al. Characterization of Patients With Ankylosing Spondylitis and Nonradiographic Axial Spondyloarthritis in the US-Based Corrona Registry. Arthritis Care Res (Hoboken). 2018;70(11):1661-70.

5. Reveille JD, Witter JP, Weisman MH. Prevalence of axial spondylarthritis in the United States: estimates from a cross-sectional survey. Arthritis Care Res (Hoboken). 2012;64(6):905-10.

6. Curtis JR, Harrold LR, Asgari MM, Deodhar A, Salman C, Gelfand JM, et al. Diagnostic Prevalence of Ankylosing Spondylitis Using Computerized Health Care Data, 1996 to 2009: Underrecognition in a US Health Care Setting. Perm J. 2016;20(4):15-151.

7. Deodhar A, Mease PJ, Reveille JD, Curtis JR, Chen S, Malhotra K, et al. Frequency of Axial Spondyloarthritis Diagnosis Among Patients Seen by US Rheumatologists for Evaluation of Chronic Back Pain. Arthritis Rheumatol. 2016;68(7):1669-76.

8. Yi E, Ahuja A, Rajput T, George AT, Park Y. Clinical, Economic, and Humanistic Burden Associated With Delayed Diagnosis of Axial Spondyloarthritis: A Systematic Review. Rheumatol Ther. 2020;7(1):65-87.

9. Barnett R, Ingram T, Sengupta R. Axial spondyloarthritis 10 years on: still looking for the lost tribe. Rheumatology (Oxford). 2020;59(Supplement_4):iv25-iv37.

10. Danve A, Deodhar A. Axial spondyloarthritis in the USA: diagnostic challenges and missed opportunities. Clin Rheumatol. 2019;38(3):625-34.

11. Rusman T, van Vollenhoven RF, van der Horst-Bruinsma IE. Gender Differences in Axial Spondyloarthritis: Women Are Not So Lucky. Curr Rheumatol Rep. 2018;20(6):35. 
12. Deodhar A, van der Heijde D, Gensler LS, Kim TH, Maksymowych WP, Ostergaard M, et al. Ixekizumab for patients with non-radiographic axial spondyloarthritis (COAST-X): a randomised, placebocontrolled trial. Lancet. 2020;395(10217):53-64.

13. Deodhar A, Blanco R, Dokoupilova E, Hall S, Kameda H, Kivitz AJ, et al. Secukinumab improves signs and symptoms of non-radiographic axial spondyloarthritis: primary results of a randomized controlled phase III study. Arthritis Rheumatol. 2020.

14. Deodhar A, Gensler LS, Kay J, Maksymowych WP, Haroon N, Landewe R, et al. A Fifty-Two-Week, Randomized, Placebo-Controlled Trial of Certolizumab Pegol in Nonradiographic Axial Spondyloarthritis. Arthritis Rheumatol. 2019;71(7):1101-11.

15. Dougados M, van der Heijde D, Sieper J, Braun J, Citera G, Lenaerts J, et al. Effects of Long-Term Etanercept Treatment on Clinical Outcomes and Objective Signs of Inflammation in Early Nonradiographic Axial Spondyloarthritis: 104-Week Results From a Randomized, Placebo-Controlled Study. Arthritis Care Res (Hoboken). 2017;69(10):1590-8.

16. van der Heijde D, Sieper J, Maksymowych WP, Lambert RG, Chen S, Hojnik M, et al. Clinical and MRI remission in patients with nonradiographic axial spondyloarthritis who received long-term open-label adalimumab treatment: 3-year results of the ABILITY-1 trial. Arthritis Res Ther. 2018;20(1):61.

17. Corbett M, Soares M, Jhuti G, Rice S, Spackman E, Sideris E, et al. Tumour necrosis factor-alpha inhibitors for ankylosing spondylitis and non-radiographic axial spondyloarthritis: a systematic review and economic evaluation. Health Technol Assess. 2016;20(9):1-334, v-vi.

18. Martindale J, Goodacre L. The journey to diagnosis in AS/axial SpA: the impact of delay. Musculoskeletal Care. 2014;12(4):221-31.

19. Deodhar A, Mittal M, Reilly P, Bao Y, Manthena S, Anderson J, et al. Ankylosing spondylitis diagnosis in US patients with back pain: identifying providers involved and factors associated with rheumatology referral delay. Clin Rheumatol. 2016;35(7):1769-76.

20. Masson Behar V, Dougados M, Etcheto A, Kreis S, Fabre S, Hudry C, et al. Diagnostic delay in axial spondyloarthritis: A cross-sectional study of 432 patients. Joint Bone Spine. 2017;84(4):467-71.

21. Redeker I, Callhoff J, Hoffmann F, Haibel H, Sieper J, Zink A, et al. Determinants of diagnostic delay in axial spondyloarthritis: an analysis based on linked claims and patient-reported survey data. Rheumatology (Oxford). 2019;58(9):1634-8.

22. Garrido-Cumbrera M, Poddubnyy D, Gossec L, Galvez-Ruiz D, Bundy C, Mahapatra R, et al. The European Map of Axial Spondyloarthritis: Capturing the Patient Perspective-an Analysis of 2846 Patients Across 13 Countries. Curr Rheumatol Rep. 2019;21(5):19.

23. Ogdie A, Benjamin Nowell W, Reynolds R, Gavigan K, Venkatachalam S, de la Cruz M, et al. RealWorld Patient Experience on the Path to Diagnosis of Ankylosing Spondylitis. Rheumatol Ther. 2019;6(2):255-67.

24. Jovani V, Blasco-Blasco M, Pascual E, Ruiz-Cantero MT. Challenges to conquer from the gender perspective in medicine: The case of spondyloarthritis. PLoS One. 2018;13(10):e0205751. 
25. Sykes MP, Doll H, Sengupta R, Gaffney K. Delay to diagnosis in axial spondyloarthritis: are we improving in the UK? Rheumatology (Oxford). 2015;54(12):2283-4.

26. Rudwaleit M, Landewe R, van der Heijde D, Listing J, Brandt J, Braun J, et al. The development of Assessment of SpondyloArthritis international Society classification criteria for axial spondyloarthritis (part I): classification of paper patients by expert opinion including uncertainty appraisal. Ann Rheum Dis. 2009;68(6):770-6.

27. Zhao SS, Pittam B, Harrison NL, Ahmed AE, Goodson NJ, Hughes DM. Diagnostic delay in axial spondyloarthritis: a systematic review and meta-analysis. Rheumatology (Oxford). 2021.

28. Bennett AN, Marzo-Ortega H, Kaur-Papadakis D, Rehman A, BritspA. The Use of Magnetic Resonance Imaging in Axial Spondyloarthritis: Time to Bridge the Gap Between Radiologists and Rheumatologists. J Rheumatol. 2017;44(6):780-5.

29. Weber U, Jurik AG, Zejden A, Larsen E, Jorgensen SH, Rufibach K, et al. Frequency and Anatomic Distribution of Magnetic Resonance Imaging Features in the Sacroiliac Joints of Young Athletes: Exploring "Background Noise" Toward a Data-Driven Definition of Sacroiliitis in Early Spondyloarthritis. Arthritis Rheumatol. 2018;70(5):736-45.

30. de Winter J, de Hooge $M$, van de Sande $M$, de Jong $H$, van Hoeven $L$, de Koning A, et al. Magnetic Resonance Imaging of the Sacroiliac Joints Indicating Sacroiliitis According to the Assessment of SpondyloArthritis international Society Definition in Healthy Individuals, Runners, and Women With Postpartum Back Pain. Arthritis Rheumatol. 2018;70(7):1042-8.

31. de Bruin F, Treyvaud MO, Feydy A, de Hooge M, Pialat JB, Dougados M, et al. Prevalence of degenerative changes and overlap with spondyloarthritis-associated lesions in the spine of patients from the DESIR cohort. RMD Open. 2018;4(1):e000657.

32. de Bruin F, ter Horst S, Bloem HL, van den Berg R, de Hooge M, van Gaalen F, et al. Prevalence of degenerative changes of the spine on magnetic resonance images and radiographs in patients aged 16-45 years with chronic back pain of short duration in the Spondyloarthritis Caught Early (SPACE) cohort. Rheumatology (Oxford). 2016;55(1):56-65.

33. Jamal M, Korver AM, Kuijper M, Lopes Barreto D, Appels CWY, Spoorenberg APL, et al. The IMPACT study: A clustered randomized controlled trial to assess the effect of a referral algorithm for axial spondyloarthritis. PLoS One. 2020;15(1):e0227025.

34. Baraliakos X, Tsiami S, Redeker I, Tsimopoulos K, Marashi A, Ruetten S, et al. Early recognition of patients with axial spondyloarthritis-evaluation of referral strategies in primary care. Rheumatology (Oxford). 2020.

35. Renson T, Carron P, De Craemer AS, Deroo L, de Hooge M, Krabbe S, et al. Axial involvement in patients with early peripheral spondyloarthritis: a prospective MRI study of sacroiliac joints and spine. Ann Rheum Dis. 2021;80(1):103-8.

36. de Winter JJ, Paramarta JE, de Jong HM, van de Sande MG, Baeten DL. Peripheral disease contributes significantly to the level of disease activity in axial spondyloarthritis. RMD Open. 2019;5(1):e000802. 
37. Swinnen TW, Vlaeyen JWS, Dankaerts W, Westhovens R, de Vlam K. Activity Limitations in Patients with Axial Spondyloarthritis: A Role for Fear of Movement and (Re)injury Beliefs. J Rheumatol. 2018;45(3):357-66.

38. Masi AT. Might axial myofascial properties and biomechanical mechanisms be relevant to ankylosing spondylitis and axial spondyloarthritis? Arthritis Res Ther. 2014;16(2):107.

39. Jacques $P, M c G o n a g l e ~ D$. The role of mechanical stress in the pathogenesis of spondyloarthritis and how to combat it. Best Pract Res Clin Rheumatol. 2014;28(5):703-10.

40. Ramiro S, Landewé R, van Tubergen A, Boonen A, Stolwijk C, Dougados M, et al. Lifestyle factors may modify the effect of disease activity on radiographic progression in patients with ankylosing spondylitis: a longitudinal analysis. RMD Open. 2015;1(1):e000153.

41. Ward MM, Reveille JD, Learch TJ, Davis JC, Jr., Weisman MH. Occupational physical activities and long-term functional and radiographic outcomes in patients with ankylosing spondylitis. Arthritis Rheum. 2008;59(6):822-32.

42. Jovani V, Blasco-Blasco M, Ruiz-Cantero MT, Pascual E. Understanding How the Diagnostic Delay of Spondyloarthritis Differs Between Women and Men: A Systematic Review and Metaanalysis. J Rheumatol. 2017;44(2):174-83.

43. Swinnen TW, Westhovens R, Dankaerts W, de Vlam K. Widespread pain in axial spondyloarthritis: clinical importance and gender differences. Arthritis Res Ther. 2018;20(1):156.

44. Templeton KJ. Sex and Gender Issues in Pain Management. J Bone Joint Surg Am. 2020;102 Suppl 1:32-5.

45. Lindstrom Egholm C, Krogh NS, Pincus T, Dreyer L, Ellingsen T, Glintborg B, et al. Discordance of Global Assessments by Patient and Physician Is Higher in Female than in Male Patients Regardless of the Physician's Sex: Data on Patients with Rheumatoid Arthritis, Axial Spondyloarthritis, and Psoriatic Arthritis from the DANBIO Registry. J Rheumatol. 2015;42(10):1781-5.

46. Samulowitz A, Gremyr I, Eriksson E, Hensing G. "Brave Men" and "Emotional Women": A TheoryGuided Literature Review on Gender Bias in Health Care and Gendered Norms towards Patients with Chronic Pain. Pain Res Manag. 2018;2018:6358624.

47. Lapane KL, Khan S, Shridharmurthy D, Beccia A, Dube C, Yi E, et al. Primary care physician perspectives on barriers to diagnosing axial Spondyloarthritis: a qualitative study. BMC Fam Pract. 2020;21(1):204.

48. 2015 Workforce Study of Rheumatology Specialists in the United States. American College of Rheumatology; 2016.

\section{Figures}




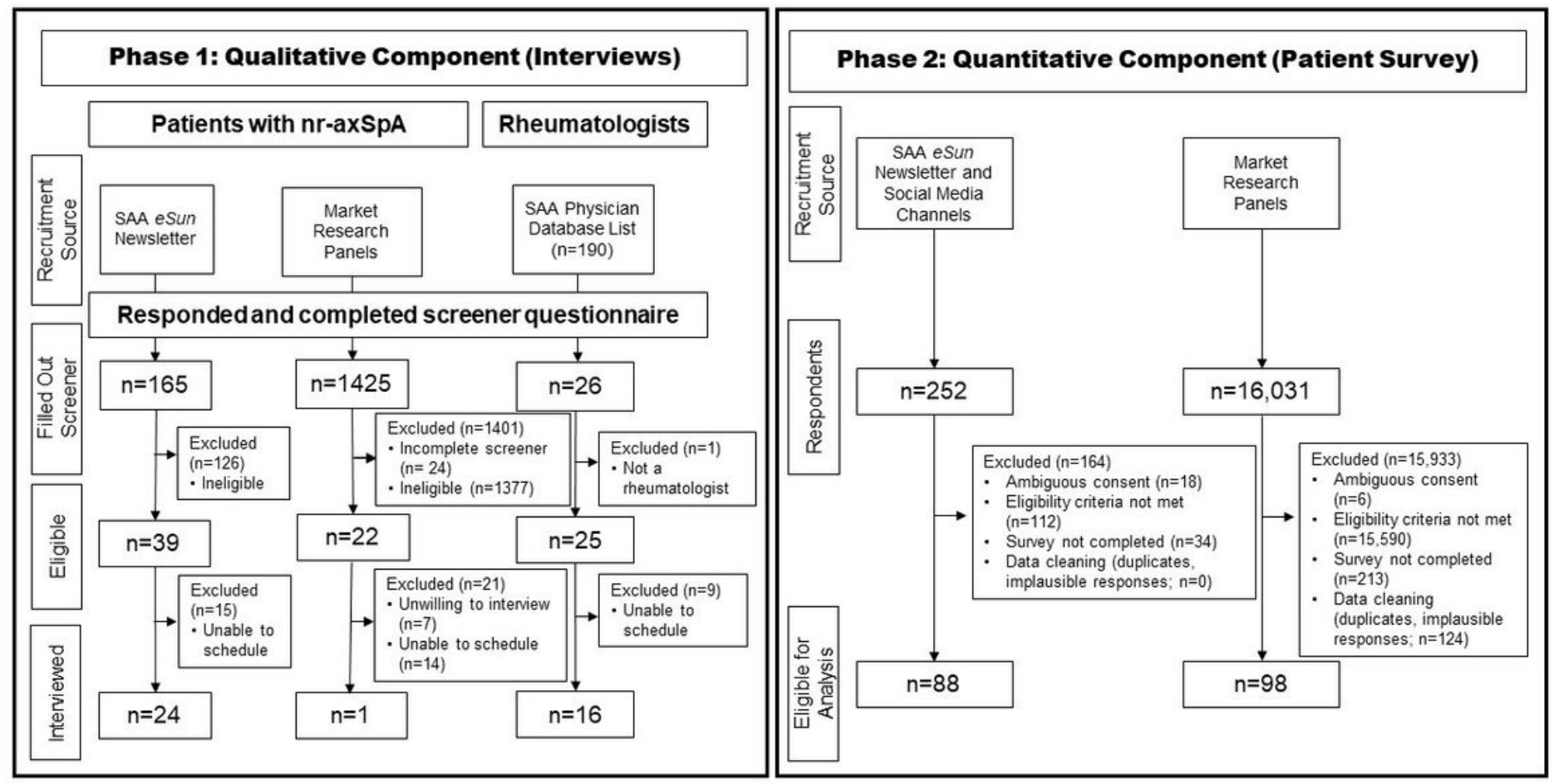

\section{Figure 1}

Participant Recruitment and Enrollment for Qualitative and Quantitative Study Components

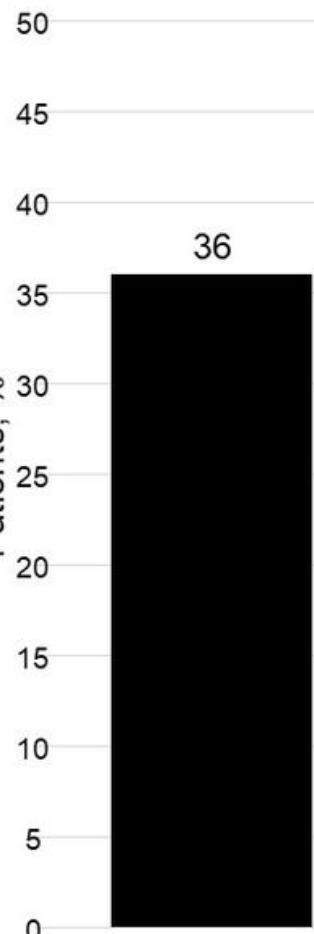

$<1$

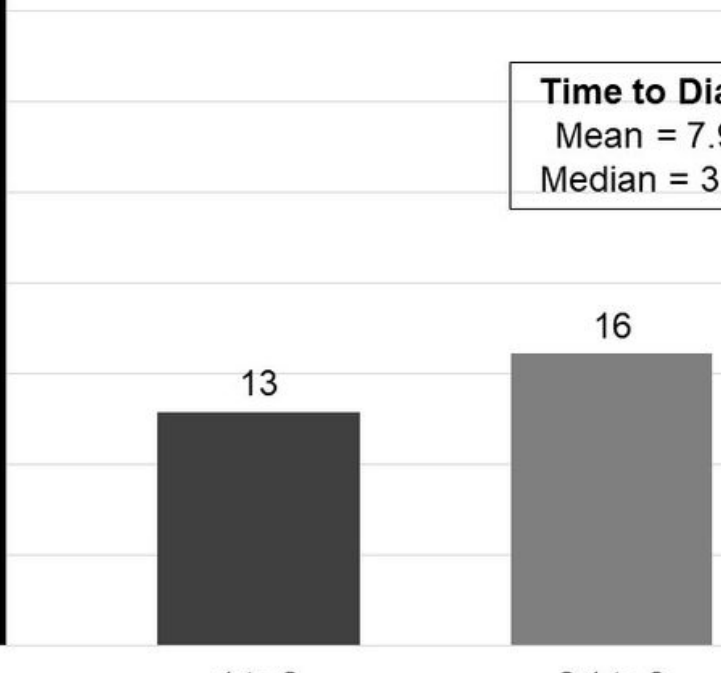

1 to 2
2.1 to 6

Time to diagnosis, years

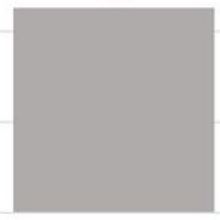

6.1 to 11 
Time to Diagnosis of nr-axSpA Footnote: a Data missing for $1 \%$ of respondents.

A

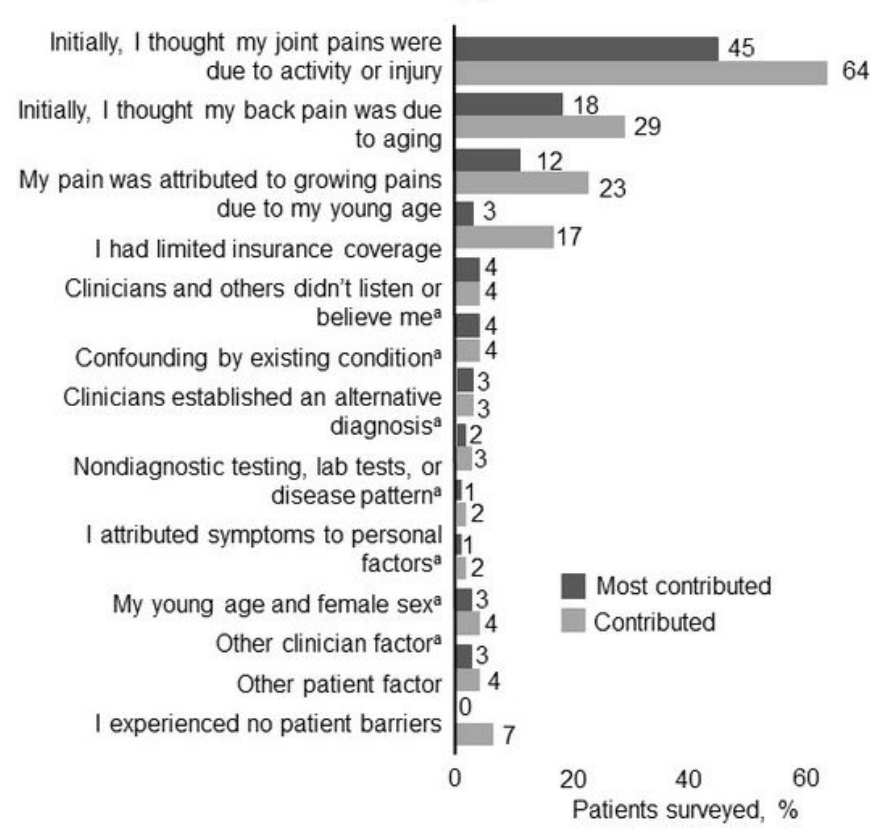

B

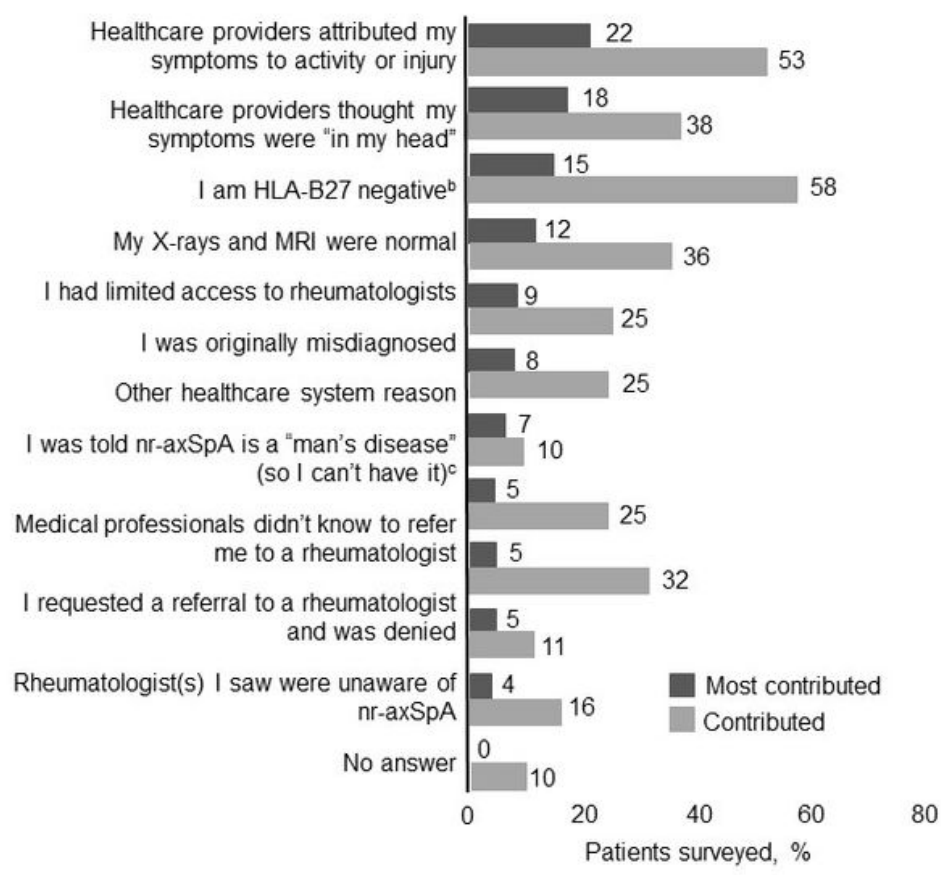

\section{Figure 3}

Factors Contributing to Prevention of Timely or Accurate nr-axSpA Diagnosis Legend: Patients were queried as to which factors were most impactful in(Most contributed), and which factors contributed to (Contributed), preventing timely or accurate nr-axSpA diagnosis. These factors were later assessed as patient-related factors (A) or healthcare provider-related factors (B). Footnotes: a Themes identified in open-ended "Other Patient Factor" responses. b Based on responses for individuals indicating HLA-B27 negative status only. c Based on responses for women only.

\section{Supplementary Files}

This is a list of supplementary files associated with this preprint. Click to download.

- Additionalfile1.docx

- Additionalfile2.docx

- Additionalfile3.pptx

- Additionalfile4.docx

- Additionalfile5.docx

- Additionalfile6.pptx

- Additionalfile7.pptx 
- Additionalfile8.pptx

- Additionalfile9.docx

- Additionalfile10.pptx 